\title{
Article \\ Common Fixed Points Technique for Existence of a Solution of Urysohn Type Integral Equations System in Complex Valued $b$-Metric Spaces
}

\author{
Muhammad Suhail Aslam 1,+, Monica Felicia Bota ${ }^{2, *,+} \mathbb{D}$, Mohammad S. R. Chowdhury ${ }^{1,+}$, Liliana Guran ${ }^{3,4,+} \mathbb{D}$ \\ and Naeem Saleem ${ }^{5,+}$ \\ 1 Department of Mathematics and Statistics, Faculty of Science, University of Lahore, Lahore 54000, Pakistan; \\ DMAT01193006@student.uol.edu.pk (M.S.A.); showkat.rahim@math.uol.edu.pk (M.S.R.C.) \\ 2 Department of Mathematics, Babeş-Bolyai University, 400084 Cluj-Napoca, Romania \\ 3 Department of Pharmaceutical Sciences, Faculty of Pharmacy, Vasile Goldiş Western University of Arad, \\ 310130 Arad, Romania; guran.liliana@uvvg.ro or liliana.guran@ulbsibiu.ro \\ 4 Department of Teacher Training, Lucian Blaga University of Sibiu, 550024 Sibiu, Romania \\ 5 Department of Mathematics, University of Management and Technology C-II, Johar Town, \\ Lahore 54782, Pakistan; naeem.saleem2@gmail.com \\ * Correspondence: bmonica@math.ubbcluj.ro \\ + These authors contributed equally to this work.
}

check for

updates

Citation: Aslam, M.S.; Bota, M.F. Mohammad, S.R.C.; Guran, L.; Saleem, N. Common Fixed Points Technique for Existence of a Solution of Urysohn Type Integral Equations System in Complex Valued $b$-Metric Spaces. Mathematics 2021, 9, 400. https://doi.org/10.3390/math9040400

Academic Editors: Simeon Reich and Temirkhan Aleroev

Received: 27 December 2020 Accepted: 7 February 2021

Published: 18 February 2021

Publisher's Note: MDPI stays neutral with regard to jurisdictional clai$\mathrm{ms}$ in published maps and institutional affiliations.

Copyright: (C) 2021 by the authors. Licensee MDPI, Basel, Switzerland. This article is an open access article distributed under the terms and conditions of the Creative Commons Attribution (CC BY) license (https:// creativecommons.org/licenses/by/ $4.0 /)$.
Abstract: In this paper we give some common fixed point theorems for Ćirić type operators in complex valued $b$-metric spaces. Also, some corollaries under this contraction condition are obtained. Our results extend and generalize the results of Hammad et al. In the second part of the paper, in order to strengthen our main results, an illustrative example and some applications are given.

Keywords: single-valued mappings; complex valued $b$-metric spaces; common fixed point; nonlinear integral equations

\section{Introduction and Preliminaries}

The Banach contraction principle is an important tool used by many authors in the field of nonlinear analysis. There exist various generalizations of the contraction principle, roughly obtained by weakening the contractive properties of the considered mapping. It can also be generalized in the sense that the working spaces will be enhanced with different structures (for example, see [1-5]).

The concept of $b$-metric space was initiated in 1989 by Bakhtin in [6] and after that Czerwik defined it as the known structure, which is considered a generalization of the ordinary metric space (see $[7,8])$. Let us recall the notion of the $b$-metric space.

Definition $1([6,8])$. Let $X$ be a non empty set and consider $s \geq 1$ a given real number. Then the following functional $d: X \times X \rightarrow[0, \infty)$ is said to be a $b$-metric if the three conditions are satisfied:

1. $d(x, y)=0$ if and only if $x=y$,

2. $d(x, y)=d(y, x)$,

3. $d(x, z) \leq s[d(x, y)+d(y, z)]$,

for all $x, y, z \in X$. A pair $(X, d)$ is called a $b$-metric space.

Example 1 ([9]). Let $(X, d)$ be a metric space and $d_{1}(x, y)=(d(x, y))^{p}$, where $p \geq 1$ is a real number. Then $\left(X, d_{1}\right)$ is a b-metric space with $s=2^{p-1}$.

From the above definition we can deduce that $b$-metric is a usual metric for $s=1$. Then the class of $b$-metric spaces is considerably larger than that of usual metric spaces. For some examples we refer to [5,7-11]. 
The complex valued metric spaces was studied in 2011 by Azam et al. [12]. They gave some fixed point results for a pair of mappings which satisfy rational inequality. It is well-known that the complex valued metric space have many applications in several branches of Mathematics as algebraic geometry, number theory, or in Physics including thermodynamics, hydrodynamics, electrical engineering, mechanical engineering. Several authors have obtained interesting and applicable results in complex valued metric spaces [13-16].

We consider $\mathbb{C}$ the set of complex numbers and let $z_{1}, z_{2} \in \mathbb{C}$ be two elements from this set. One can define the following partial order $\precsim$ on $\mathbb{C}$, called in literature as lexicographic order, as follows:

$z_{1} \precsim z_{2}$ if and only if $\operatorname{Re}\left(z_{1}\right) \leq \operatorname{Re}\left(z_{2}\right)$ or $\left(\operatorname{Re}\left(z_{1}\right)=\operatorname{Re}\left(z_{2}\right)\right.$ and $\left.\operatorname{Im}\left(z_{1}\right) \leq \operatorname{Im}\left(z_{2}\right)\right)$.

Taking into account the previous definition, we have that $z_{1} \precsim z_{2}$ if one of the next conditions is satisfied:

$\left(C_{1}\right) \operatorname{Re}\left(z_{1}\right)<\operatorname{Re}\left(z_{2}\right)$ and $\operatorname{Im}\left(z_{1}\right)<\operatorname{Im}\left(z_{2}\right)$;

$\left(C_{2}\right) \operatorname{Re}\left(z_{1}\right)<\operatorname{Re}\left(z_{2}\right)$ and $\operatorname{Im}\left(z_{1}\right)=\operatorname{Im}\left(z_{2}\right)$;

$\left(C_{3}\right) \operatorname{Re}\left(z_{1}\right)<\operatorname{Re}\left(z_{2}\right)$ and $\operatorname{Im}\left(z_{1}\right)>\operatorname{Im}\left(z_{2}\right)$;

$\left(C_{4}\right) \operatorname{Re}\left(z_{1}\right)=\operatorname{Re}\left(z_{2}\right)$ and $\operatorname{Im}\left(z_{1}\right)<\operatorname{Im}\left(z_{2}\right)$.

We recall the definition of "max" function with respect to the partial order $\precsim$ in $\mathbb{C}$ gave by R.K. Varma and H.K. Pathak in [17].

Definition 2 ([17]). Let $z_{1}, z_{2} \in \mathbb{C}$. The "max" function with respect to the partial order relation $\precsim$ is defined on $\mathbb{C}$ by:

(i) $\max \left\{z_{1}, z_{2}\right\} \precsim z_{2} \Leftrightarrow z_{1} \precsim z_{2}$;

(ii) $z_{1} \precsim \max \left\{z_{2}, z_{3}\right\} \Rightarrow z_{1} \precsim z_{2}$ or $z_{1} \precsim z_{3}$;

(iii) $\max \left\{z_{1}, z_{2}\right\}=z_{2} \Leftrightarrow z_{1} \precsim z_{2}$ or $\left|z_{1}\right| \leq\left|z_{2}\right|$.

Another result useful in the proof of our main results is the following lemma.

Lemma 1 ([17]). Let $z_{1}, z_{2}, z_{3} \ldots \in \mathbb{C}$ and the partial order relation $\precsim$ is defined on $\mathbb{C}$. Then, the following statements holds:

(i) If $z_{1} \precsim \max \left\{z_{2}, z_{3}\right\}$, then $z_{1} \precsim z_{2}$ if $z_{3} \precsim z_{2}$;

(ii) If $z_{1} \precsim \max \left\{z_{2}, z_{3}, z_{4}\right\}$, then $z_{1} \precsim z_{2}$ if $\max \left\{z_{3}, z_{4}\right\} \precsim z_{2}$;

(iii) If $z_{1} \precsim \max \left\{z_{2}, z_{3}, z_{4}, z_{5}\right\}$, then $z_{1} \precsim z_{2}$ if $\max \left\{z_{3}, z_{4}, z_{5}\right\} \precsim z_{2}$ and so on.

In 2011 Azam et al. gave in [12] the definition of the complex valued metric as follows.

Definition 3 ([12]). Let $X$ be a nonempty set. A mapping $d: X \times X \rightarrow \mathbb{C}$ is called a complex valued metric on $X$ if, for all $x, y, z \in X$, the following conditions hold:

$\left(C M_{1}\right) 0 \precsim d(x, y)$ and $d(x, y)=0$ if and only if $x=y$,

$\left(C M_{2}\right) d(x, y)=d(y, x)$,

$\left(C M_{3}\right) d(x, y) \precsim d(x, z)+d(z, y)$.

Then $d$ is called a complex valued metric on $X$ and $(X, d)$ is called a complex valued metric space.

For some examples in this space we refer to $[2,13,15,17-20]$.

In 2013, Rao et al. (see [21]) gave the definition of complex valued $b$-metric space, which is more general than the notion of complex valued metric space introduced by Azam et al. in 2011. In the area of $b$-metric spaces, one can find in the literature a lot of articles which deal with fixed point theory and it's applications (for example, see $[6,8,9,13,15,16,20-23]$ ).

Definition 4 ([21]). Let $X$ be a nonempty set. Consider $s \geq 1$ a given real number, then a mapping $d: X \times X \rightarrow \mathbb{C}$ is called a complex valued b-metric on $X$ if the next conditions hold, for all 
$x, y, z \in X:$

$\left(C B M_{1}\right) 0 \precsim d(x, y)$ and $d(x, y)=0$ if and only if $x=y$,

$\left(C B M_{2}\right) d(x, y)=d(y, x)$,

$\left(C B M_{3}\right) d(x, y) \precsim s[d(x, z)+d(z, y)]$.

Then $d$ is called a complex valued $b$-metric on $X$ and $(X, d)$ is called a complex valued b-metric space.

Next, let us recall some properties for complex valued $b$-metric spaces.

Definition 5 ([21]). Let $(X, d)$ be a complex valued $b$-metric space and $\left\{x_{n}\right\}$ a sequence in $X$ and $x \in X$. Consider the following.

(i) A sequence $\left\{x_{n}\right\}$ in $X$ is said to be convergent to $x \in X$ if for every $0 \prec c \in \mathbb{C}$ there exists $N \in \mathbb{N}$ such that $d\left(x_{n}, x\right) \prec c$ for every $n>N$. We denote this by $\lim _{n \rightarrow \infty} x_{n}=x$ or $x_{n} \rightarrow x$ as $n \rightarrow \infty$.

(ii) If for every $0 \prec c \in \mathbb{C}$ there exists $N \in \mathbb{N}$ such that $d\left(x_{n}, x_{n+m}\right) \prec c$ for every $n>N$ and $m \in N$. Then $\left\{x_{n}\right\}$ is called a Cauchy sequence in $(X, d)$.

(iii) If every Cauchy sequence in $X$ is convergent in $X$ then $(X, d)$ is called a complete complex valued b-metric space.

Lemma 2 ([21]). Let $(X, d)$ be a complex valued b-metric space and $\left\{x_{n}\right\}$ be a sequence in $X$. Then $\left\{x_{n}\right\}$ converges to $x$ if and only if $\left|d\left(x_{n}, x\right)\right| \rightarrow 0$ as $n \rightarrow \infty$.

Lemma 3 ([21]). Let $(X, d)$ be a complex valued $b$-metric space. Then a sequence $\left\{x_{n}\right\}$ in $X$ is a Cauchy sequence if and only if $\left|d\left(x_{n}, x_{n+m}\right)\right| \rightarrow 0$ as $n \rightarrow \infty$ where $m \in N$.

Further, we recall the following generalization of the contraction principle given by $\mathrm{Lj}$. Ćirić in [24].

Theorem 1. Let $(X, d)$ be a metric space and $T: X \rightarrow X$ be a mapping for which there exists $q \in(0,1)$ such that, for all $x, y \in X$, we have

$$
d(T x, T y) \leq q \max \left\{d(x, y), d(x, T x), d(y, T y), \frac{1}{2}(d(x, T y)+d(y, T x))\right\},
$$

If $X$ is T-orbitally complete then:

1. $\operatorname{Fix}(T)=\left\{x^{*}\right\}$;

2. for every $x \in X$ the sequence $\left(T^{n} x\right)_{n \in \mathbb{N}}$ converges to $x^{*}$;

3. $d\left(T^{n} x, x^{*}\right) \leq \frac{q^{n}}{1-q} d(x, T x)$, for all $x \in X$.

Throughout this paper $\mathbb{N}$ will be the set of all natural numbers and we will use $b$ CVMS as a notation for complex valued $b$-metric space. Also, by $\mathbb{N}^{*}:=\mathbb{N}-\{0\}$ we denote the set of all natural numbers without 0 . A well known notation is that of the set of fixed point for an operator. In our case, $\operatorname{Fix}(T):=\{x \in X \mid x \in T(x)\}$.

In this paper we prove some fixed point theorems in complex valued metric spaces. The mappings satisfy some Ćirić type contractive conditions. Moreover, we give some examples and applications which will strengthen our results.

\section{Common Fixed Points for Ćirić Type Operators}

First of all let us give some lemmas for the case of complex valued $b$-metric spaces, which will be useful in proving our first common fixed point result. 
Lemma 4. For each sequence $\left(x_{n}\right)_{n \in \mathbb{N}}$ of elements from a complex valued $b$-metric space $(X, d)$, the inequality

$$
d\left(x_{0}, x_{k}\right) \precsim s^{n} \sum_{k=1}^{i=0} d\left(x_{i}, x_{i+1}\right)
$$

holds for each $n \in \mathbb{N}$ and each $k \in\left\{1,2,3, \ldots, 2^{n-1}, 2^{n}\right\}$.

Lemma 5. Every sequence $\left(x_{n}\right)_{n \in \mathbb{N}}$ of elements from a complex valued b-metric space $(X, d)$ with constant s having the property that there exists $\gamma \in[0,1)$ such that $d\left(x_{n+1}, x_{n}\right) \precsim \gamma d\left(x_{n}, x_{n-1}\right)$, $n \in \mathbb{N}$ is a Cauchy sequence. Moreover, the following estimation holds

$$
d\left(x_{n+1}, x_{n+p}\right) \precsim \frac{\gamma^{n} S}{1-\gamma} d\left(x_{0}, x_{1}\right), \text { for all } n, p \in \mathbb{N},
$$

where $S:=\sum_{i=1}^{\infty} \gamma^{2 i \log _{\gamma} s+2^{i-1}}$.

Remark 1. Following the same steps as in the proof of Lemma 4 and Lemma 5 given for the case of b-metric spaces by Miculescu R. and Mihail A. in [25], we get same conclusions for the case of complex valued b-metric spaces.

Now, we present a new common fixed point result for Ćirić type operators in the case of complex valued $b$-metric spaces.

Theorem 2. Let $(X, d)$ be a complete $b$-CVMS with the coefficient $s \geq 1$ and $S, T: X \rightarrow X$ be two continuous mappings such that

$$
d(S x, T y) \precsim q \max \left\{d(x, y), d(x, S x), d(y, T y), \frac{1}{2}(d(x, T y)+d(y, S x))\right\},
$$

for all $x, y \in X$, where $0 \leq q<\frac{1}{s}$ and all elements on the right side are comparable to each other w.r.t. the partial order $\precsim$. Then the pair $(S, T)$ has a unique common fixed point.

Proof. Let $x_{0}$ be arbitrary point in $X$ and define a sequence $\left\{x_{n}\right\}$ as follows

$$
x_{2 n+1}=S x_{2 n} \text { and } x_{2 n+2}=T x_{2 n+1}, n=0,1,2, \ldots
$$

Then, by (1) and (2), we get

$$
\begin{aligned}
d\left(x_{2 n+1}, x_{2 n+2}\right)= & d\left(S x_{2 n}, T x_{2 n+1}\right) \\
\precsim & q \max \left\{d\left(x_{2 n}, x_{2 n+1}\right), d\left(x_{2 n}, S x_{2 n}\right), d\left(x_{2 n+1}, T x_{2 n+1}\right)\right. \\
& \left.\frac{1}{2}\left(d\left(x_{2 n}, T x_{2 n+1}\right)+d\left(x_{2 n+1}, S x_{2 n}\right)\right)\right\} \\
& \precsim q \max \left\{d\left(x_{2 n}, x_{2 n+1}\right), d\left(x_{2 n}, x_{2 n+1}\right), d\left(x_{2 n+1}, x_{2 n+2}\right)\right. \\
& \left.\frac{1}{2}\left(d\left(x_{2 n}, x_{2 n+2}\right)+d\left(x_{2 n+1}, x_{2 n+1}\right)\right)\right\} .
\end{aligned}
$$

\section{Case I.}

If $\max \left\{d\left(x_{2 n}, x_{2 n+1}\right), d\left(x_{2 n+1}, x_{2 n+2}\right), \frac{1}{2}\left(d\left(x_{2 n}, x_{2 n+2}\right)+d\left(x_{2 n+1}, x_{2 n+1}\right)\right)\right\}=d\left(x_{2 n+1}, x_{2 n+2}\right)$ we have:

$$
d\left(x_{2 n+1}, x_{2 n+2}\right) \precsim q d\left(x_{2 n+1}, x_{2 n+2}\right) .
$$

This implies $q \geq 1$, which is a contradiction. 


\section{Case II.}

If $\max \left\{d\left(x_{2 n}, x_{2 n+1}\right), d\left(x_{2 n+1}, x_{2 n+2}\right), \frac{1}{2}\left(d\left(x_{2 n}, x_{2 n+2}\right)+d\left(x_{2 n+1}, x_{2 n+1}\right)\right)\right\}=d\left(x_{2 n}, x_{2 n+1}\right)$ we have

$$
d\left(x_{2 n+1}, x_{2 n+2}\right) \precsim q d\left(x_{2 n}, x_{2 n+1}\right) .
$$

For the next step we have

$$
\begin{gathered}
d\left(x_{2 n+2}, x_{2 n+3}\right) \precsim q \max \left\{d\left(x_{2 n+1}, x_{2 n+2}\right), d\left(x_{2 n+2}, x_{2 n+3}\right),\right. \\
\left.\frac{1}{2}\left(d\left(x_{2 n+1}, x_{2 n+3}\right)+d\left(x_{2 n+2}, x_{2 n+2}\right)\right)\right\} .
\end{gathered}
$$

Then we have the following three cases

\section{Case IIa.}

$d\left(x_{2 n+2}, x_{2 n+3}\right) \precsim q d\left(x_{2 n+2}, x_{2 n+3}\right)$, which implies $q \geq 1$. Contradiction.

\section{Case IIb.}

$$
d\left(x_{2 n+2}, x_{2 n+3}\right) \precsim q d\left(x_{2 n+1}, x_{2 n+2}\right) .
$$

From (3) and (4), for all $n=0,1,2, \ldots$, we get

$$
d\left(x_{n+1}, x_{n+2}\right) \precsim q d\left(x_{n}, x_{n+1}\right) \precsim \ldots \precsim q^{n+1} d\left(x_{0}, x_{1}\right) .
$$

For $m, n \in \mathbb{N}$, with $m>n$, we have

$$
\begin{aligned}
d\left(x_{n}, x_{m}\right) & \precsim s\left[d\left(x_{n}, x_{n+1}\right)+d\left(x_{n+1}, x_{m}\right)\right] \\
& \precsim s\left(d\left(x_{n}, x_{n+1}\right)\right)+s^{2}\left[d\left(x_{n+1}, x_{n+2}\right)+d\left(x_{n+2}, x_{m}\right]\right. \\
& \precsim s\left(d\left(x_{n}, x_{n+1}\right)\right)+s^{2}\left(d\left(x_{n+1}, x_{n+2}\right)\right)+s^{3}\left(d\left(x_{n+2}, x_{n+3}\right)\right)+ \\
& +\ldots+s^{m-n-1}\left(d\left(x_{m-2}, x_{m-1}\right)\right)+s^{m-n}\left(d\left(x_{m-1}, x_{m}\right) .\right.
\end{aligned}
$$

Moreover, using (21) we have

$$
\begin{aligned}
d\left(x_{n}, x_{m}\right) & \precsim s q^{n}\left(d\left(x_{0}, x_{1}\right)\right)+s^{2} q^{n+1}\left(d\left(x_{0}, x_{1}\right)\right)+s^{3} q^{n+2}\left(d\left(x_{0}, x_{1}\right)\right) \\
& +\ldots+s^{m-n-1} q^{m-2}\left(d\left(x_{0}, x_{1}\right)\right)+s^{m-n} q^{m-1}\left(d\left(x_{0}, x_{1}\right)\right) \\
& =\sum_{i=1}^{m-n} s^{i} q^{i+n-1}\left(d\left(x_{0}, x_{1}\right)\right) .
\end{aligned}
$$

Therefore,

$$
\begin{aligned}
\left|d\left(x_{n}, x_{m}\right)\right| & \precsim \sum_{i=1}^{m-n} s^{i+n-1} q^{i+n-1}\left|d\left(x_{0}, x_{1}\right)\right|=\sum_{t=n}^{m-1} s^{t} q^{t}\left|d\left(x_{0}, x_{1}\right)\right| \\
& \precsim \sum_{t=n}^{\infty}(s q)^{t}\left|d\left(x_{0}, x_{1}\right)\right|=\frac{(s q)^{n}}{1-s q}\left|d\left(x_{0}, x_{1}\right)\right| .
\end{aligned}
$$

Then we have

$$
\left|d\left(x_{n}, x_{m}\right)\right| \precsim \frac{(s q)^{n}}{1-s q}\left|d\left(x_{0}, x_{1}\right)\right| \rightarrow 0 \text { as } n \rightarrow \infty .
$$

Therefore $\left\{x_{n}\right\}$ is a Cauchy sequence in $X$.

\section{Case IIc.}

$$
d\left(x_{2 n+2}, x_{2 n+3}\right) \precsim q \frac{1}{2}\left(d\left(x_{2 n+1}, x_{2 n+3}\right)+d\left(x_{2 n+2}, x_{2 n+2}\right)\right) .
$$




$$
\begin{aligned}
\frac{1}{2}\left(d\left(x_{2 n+1}, x_{2 n+3}\right)+d\left(x_{2 n+2}, x_{2 n+2}\right)\right) & =\frac{1}{2}\left(d\left(x_{2 n+1}, x_{2 n+3}\right)+0\right) \\
& \precsim \frac{s}{2}\left(d\left(x_{2 n+1}, x_{2 n+2}\right)+d\left(x_{2 n+2}, x_{2 n+3}\right)\right) .
\end{aligned}
$$

In this case we obtain

$$
d\left(x_{2 n+2}, x_{2 n+3}\right) \precsim \frac{q s}{2}\left(d\left(x_{2 n+1}, x_{2 n+2}\right)+d\left(x_{2 n+2}, x_{2 n+3}\right)\right) .
$$

Then $\left(1-\frac{q s}{2}\right) d\left(x_{2 n+2}, x_{2 n+3}\right) \precsim \frac{q s}{2} d\left(x_{2 n+1}, x_{2 n+2}\right)$.

$$
d\left(x_{2 n+1}, x_{2 n+2}\right) \precsim \frac{q s}{2}\left(d\left(x_{2 n}, x_{2 n+1}\right)+d\left(x_{2 n+1}, x_{2 n+2}\right)\right) .
$$

Then $\left(1-\frac{q s}{2}\right) d\left(x_{2 n+2}, x_{2 n+3}\right) \precsim \frac{q s}{2} d\left(x_{2 n+1}, x_{2 n+2}\right)$.

Then we get

$$
d\left(x_{2 n+2}, x_{2 n+3}\right) \precsim \frac{q s}{2-q s} d\left(x_{2 n+1}, x_{2 n+2}\right) .
$$

Then by (3) and (5) results $d\left(x_{n+1}, x_{n+2}\right) \precsim \gamma d\left(x_{n}, x_{n+1}\right)$ where $\gamma=\max \left\{q, \frac{q s}{2-q s}\right\}<1$. Applying Lemma 5 we obtain the sequence $\left(x_{n}\right)_{n \in \mathbb{N}}$ is Cauchy.

\section{Case III.}

If $\max \left\{d\left(x_{2 n}, x_{2 n+1}\right), d\left(x_{2 n+1}, x_{2 n+2}\right), \frac{1}{2}\left(d\left(x_{2 n}, x_{2 n+2}\right)+d\left(x_{2 n+1}, x_{2 n+1}\right)\right)\right\}$ $=\frac{1}{2}\left(d\left(x_{2 n}, x_{2 n+2}\right)+d\left(x_{2 n+1}, x_{2 n+1}\right)\right)$ we have

$$
\begin{aligned}
\frac{1}{2}\left(d\left(x_{2 n}, x_{2 n+2}\right)+d\left(x_{2 n+1}, x_{2 n+1}\right)\right) & =\frac{1}{2}\left(d\left(x_{2 n}, x_{2 n+2}\right)+0\right) \\
& \precsim \frac{s}{2}\left(d\left(x_{2 n}, x_{2 n+1}\right)+d\left(x_{2 n+1}, x_{2 n+2}\right)\right) .
\end{aligned}
$$

In this case we obtain

$$
d\left(x_{2 n+1}, x_{2 n+2}\right) \precsim \frac{q s}{2}\left(d\left(x_{2 n}, x_{2 n+1}\right)+d\left(x_{2 n+1}, x_{2 n+2}\right)\right) .
$$

Then $\left(1-\frac{q s}{2}\right) d\left(x_{2 n+1}, x_{2 n+2}\right) \precsim \frac{q s}{2} d\left(x_{2 n}, x_{2 n+1}\right)$.

Then we get

$$
d\left(x_{2 n+1}, x_{2 n+2}\right) \precsim \frac{q s}{2-q s} d\left(x_{2 n}, x_{2 n+1}\right) .
$$

For the next step we get

$$
\begin{gathered}
d\left(x_{2 n+2}, x_{2 n+3}\right) \precsim q \max \left\{d\left(x_{2 n+1}, x_{2 n+2}\right), d\left(x_{2 n+2}, x_{2 n+3}\right),\right. \\
\left.\frac{1}{2}\left(d\left(x_{2 n+1}, x_{2 n+3}\right)+d\left(x_{2 n+2}, x_{2 n+2}\right)\right)\right\} .
\end{gathered}
$$

Then we find three cases.

\section{Case IIIa.}

$$
d\left(x_{2 n+2}, x_{2 n+3}\right) \precsim q d\left(x_{2 n+2}, x_{2 n+3}\right) \text {, which implies } q \geq 1 \text {. Contradiction. }
$$

\section{Case IIIb.}

$$
d\left(x_{2 n+2}, x_{2 n+3}\right) \precsim q d\left(x_{2 n+1}, x_{2 n+2}\right) .
$$

Then by (6) and (7) results $d\left(x_{n+1}, x_{n+2}\right) \precsim \gamma d\left(x_{n}, x_{n+1}\right)$ where $\gamma=\max \left\{q, \frac{q s}{2-q s}\right\}<1$. Applying Lemma 5 we obtain the sequence $\left(x_{n}\right)_{n \in \mathbb{N}}$ is Cauchy. 


\section{Case IIIc.}

$$
d\left(x_{2 n+2}, x_{2 n+3}\right) \precsim q \frac{1}{2}\left(d\left(x_{2 n+1}, x_{2 n+3}\right)+d\left(x_{2 n+2}, x_{2 n+2}\right)\right) .
$$

After simple calculations we get

$$
d\left(x_{2 n+2}, x_{2 n+3}\right) \precsim \frac{q s}{2-q s} d\left(x_{2 n+1}, x_{2 n+2}\right) .
$$

Then by (6) and (8) results

$$
d\left(x_{n+1}, x_{n+2}\right) \precsim \tau d\left(x_{n}, x_{n+1}\right) \text { where } 0 \leq \tau=\frac{q s}{2-q s}<1 .
$$

Then for all $n=0,1,2, \ldots$ we get

$$
d\left(x_{n+1}, x_{n+2}\right) \precsim \tau d\left(x_{n}, x_{n+1}\right) \precsim \ldots \precsim \tau^{n+1} d\left(x_{0}, x_{1}\right) .
$$

For $m, n \in \mathbb{N}$, with $m>n$, we have

$$
\begin{aligned}
d\left(x_{n}, x_{m}\right) & \precsim s\left[d\left(x_{n}, x_{n+1}\right)+d\left(x_{n+1}, x_{m}\right)\right] \\
& \precsim s\left(d\left(x_{n}, x_{n+1}\right)\right)+s^{2}\left[d\left(x_{n+1}, x_{n+2}\right)+d\left(x_{n+2}, x_{m}\right]\right. \\
& \precsim s\left(d\left(x_{n}, x_{n+1}\right)\right)+s^{2}\left(d\left(x_{n+1}, x_{n+2}\right)\right)+s^{3}\left(d\left(x_{n+2}, x_{n+3}\right)\right)+ \\
& +\ldots+s^{m-n-1}\left(d\left(x_{m-2}, x_{m-1}\right)\right)+s^{m-n}\left(d\left(x_{m-1}, x_{m}\right)\right) .
\end{aligned}
$$

Using (9) we obtain

$$
\begin{aligned}
d\left(x_{n}, x_{m}\right) & \precsim s \tau^{n}\left(d\left(x_{0}, x_{1}\right)\right)+s^{2} \tau^{n+1}\left(d\left(x_{0}, x_{1}\right)\right)+s^{3} \tau^{n+2}\left(d\left(x_{0}, x_{1}\right)\right) \\
& +\ldots+s^{m-n-1} \tau^{m-2}\left(d\left(x_{0}, x_{1}\right)\right)+s^{m-n} \tau^{m-1}\left(d\left(x_{0}, x_{1}\right)\right) \\
& =\sum_{i=1}^{m-n} s^{i} \tau^{i+n-1}\left(d\left(x_{0}, x_{1}\right)\right) .
\end{aligned}
$$

Therefore,

$$
\begin{aligned}
\left|d\left(x_{n}, x_{m}\right)\right| & \precsim \sum_{i=1}^{m-n} s^{i+n-1} \tau^{i+n-1}\left|d\left(x_{0}, x_{1}\right)\right|=\sum_{t=n}^{m-1} s^{t} \tau^{t}\left|d\left(x_{0}, x_{1}\right)\right| \\
& \precsim \sum_{t=n}^{\infty}(s \tau)^{t}\left|d\left(x_{0}, x_{1}\right)\right|=\frac{(s \tau)^{n}}{1-s q}\left|d\left(x_{0}, x_{1}\right)\right| .
\end{aligned}
$$

Then we have

$$
\left|d\left(x_{n}, x_{m}\right)\right| \precsim \frac{(s \tau)^{n}}{1-s \tau}\left|d\left(x_{0}, x_{1}\right)\right| \rightarrow 0 \text { as } n \rightarrow \infty .
$$

Therefore $\left\{x_{n}\right\}$ is a Cauchy sequence in $X$.

In all the cases above discussed we get the sequence $\left(x_{n}\right)_{n \in \mathbb{N}}$ is a Cauchy sequence. Since $X$ is complete there exists $x^{*} \in X$ such that $d\left(x_{n}, x^{*}\right) \rightarrow 0$ as $n \rightarrow \infty$. Then result $d\left(x_{2 n}, x^{*}\right) \rightarrow 0$ as $n \rightarrow \infty$. By the continuity of $S$ it follows $x_{2 n+1}=S x_{2 n} \rightarrow S x^{*}$ as $n \rightarrow \infty$. By the uniqueness of the limit we get that $x^{*}=S x^{*}$.

In the same time, we have $d\left(x_{2 n+1}, x^{*}\right) \rightarrow 0$ as $n \rightarrow \infty$. By the continuity of $T$ it follows $x_{2 n+2}=T x_{2 n+1} \rightarrow T x^{*}$ as $n \rightarrow \infty$. By the uniqueness of the limit we get that $x^{*}=T x^{*}$.

Then $x^{*}$ is a common fixed point of the pair $(S, T)$. 
For uniqueness, assume that $y^{*} \in X$ is another common fixed point for the pair $(S, T)$. Then

$$
\begin{aligned}
d\left(x^{*}, y^{*}\right) & =d\left(S x^{*}, T y^{*}\right) \\
& \precsim q \max \left\{d\left(x^{*}, y^{*}\right), d\left(x^{*}, S x^{*}\right), d\left(y^{*}, T y^{*}\right), \frac{1}{2}\left(d\left(x^{*}, T y^{*}\right)+d\left(y^{*}, S x^{*}\right)\right)\right\} \\
& \precsim q \max \left\{d\left(x^{*}, y^{*}\right), d\left(x^{*}, x^{*}\right), d\left(y^{*}, y^{*}\right), \frac{1}{2}\left(d\left(x^{*}, y^{*}\right)+d\left(y^{*}, x^{*}\right)\right)\right\} . \\
& \precsim q d\left(x^{*}, y^{*}\right) .
\end{aligned}
$$

This implies that $x^{*}=y^{*}$, which completes the proof.

If we omit the continuity condition for the mappings $S$ and $T$ we get the following result.

Theorem 3. Let $(X, d)$ be a complete $b$-CVMS with the coefficient $s \geq 1$ and $S, T: X \rightarrow X$ be two mappings such that

$$
d(S x, T y) \precsim q \max \left\{d(x, y), d(x, S x), d(y, T y), \frac{1}{2}(d(x, T y)+d(y, S x))\right\},
$$

for all $x, y \in X$, where $0 \leq q<\frac{1}{S}$. Then the pair $(S, T)$ has a unique common fixed point.

Proof. Following same steps as in the proof of Theorem 2 we obtain that the sequence $\left\{x_{n}\right\}$ is a Cauchy sequence. Since $X$ is complete there exists $x^{*} \in X$ such that $d\left(x_{n}, x^{*}\right) \rightarrow 0$ as $n \rightarrow \infty$.

Since $S$ and $T$ are not continuous we have $d\left(x^{*}, S x^{*}\right)=z>0$. Then we estimate

$$
\begin{aligned}
z=d\left(x^{*}, S x^{*}\right) & \precsim s\left[d\left(x^{*}, x_{2 k+2}\right)+d\left(x_{2 k+2}, S x^{*}\right)\right] \\
& \precsim s d\left(x^{*}, x_{2 k+2}\right)+s d\left(T x_{2 k+1}, S x^{*}\right) \\
& \precsim s d\left(x^{*}, x_{2 k+2}\right)+s q \max \left\{d\left(x_{2 k+1}, x^{*}\right), d\left(x_{2 k+1}, T x_{2 k+1}\right), d\left(x^{*}, S x^{*}\right),\right. \\
& \left.\frac{1}{2}\left(d\left(x_{2 k+1}, S x^{*}\right)+d\left(x^{*}, T x_{2 k+1}\right)\right)\right\} \\
& \precsim s d\left(x^{*}, x_{2 k+2}\right)+s q \max \left\{d\left(x_{2 k+1}, x^{*}\right), d\left(x_{2 k+1}, x_{2 k+2}\right) d\left(x^{*}, S x^{*}\right),\right. \\
& \left.\frac{1}{2}\left(d\left(x_{2 k+1}, S x^{*}\right)+d\left(x^{*}, x_{2 k+2}\right)\right)\right\} \\
& \precsim s d\left(x^{*}, x_{2 k+2}\right)+s q d\left(x^{*}, S x^{*}\right) \precsim s d\left(x^{*}, x_{k+2}\right)+s q z .
\end{aligned}
$$

This yields,

$$
|z| \leq s\left|d\left(x^{*}, x_{2 k+2}\right)\right|+s q|z|
$$

Then results $q \geq 1$ which is a contradiction. Then $x^{*}=S x^{*}$. In the same way we get $x^{*}=T x^{*}$. Hence $x^{*}$ is a common fixed point for the pair $(S, T)$. For uniqueness of the common fixed point $x^{*}$ we use similar steps as in the proof of Theorem 2.

For $S=T$ we get the following fixed points result for Ćirić type operators on complex valued $b$-metric space.

Theorem 4. Let $(X, d)$ be a complete $b$-CVMS with the coefficient $s \geq 1$ and $S: X \rightarrow X$ be $a$ continuous mapping which satisfy

$$
d(S x, S y) \precsim q \max \left\{d(x, y), d(x, S x), d(y, S y), \frac{1}{2}(d(x, S y)+d(y, S x))\right\},
$$

for all $x, y \in X$, where $0 \leq q<\frac{1}{s}$. Then $T$ have a unique fixed point.

Remark 2. We get a similar fixed point result if we omit the continuity condition for the operator S. 
Now we obtain the following fixed point result.

Corollary 1. Let $(X, d)$ be a complete $b$-CVMS with $s \geq 1$ be a given real number and $T: X \rightarrow X$ a continuous mapping which satisfies

$$
d\left(T^{n} x, T^{n} y\right) \precsim q \max \left\{d(x, y), d\left(x, T^{n} x\right), d\left(y, T^{n} y\right), \frac{1}{2}\left(d\left(x, T^{n} y\right)+d\left(y, T^{n} x\right)\right)\right\},
$$

for all $x, y \in X$, where $0 \leq q<\frac{1}{s}, n \in \mathbb{N}$ and all elements on the right side are comparable to each other w.r.t. the partial order $\precsim$. Then $T$ has a unique fixed point.

Proof. By Theorem 2 we obtain $x^{*} \in X$ such that

$$
T^{n} x^{*}=x^{*} .
$$

Then we get:

$$
\begin{aligned}
d\left(T x^{*}, x^{*}\right)= & d\left(T T^{n} x^{*}, T^{n} x^{*}\right)=d\left(T^{n} T x^{*}, T^{n} x^{*}\right) \\
\precsim & q \max \left\{d\left(T x^{*}, x^{*}\right), d\left(T x^{*}, T^{n} T x^{*}\right), d\left(x^{*}, T^{n} x^{*}\right),\right. \\
& \left.\frac{1}{2}\left(d\left(T x^{*}, T^{n} x^{*}\right)+d\left(x^{*}, T^{n} T x^{*}\right)\right)\right\} \\
\precsim & q \max \left\{d\left(T x^{*}, x^{*}\right), d\left(T x^{*}, T x^{*}\right), d\left(x^{*}, x^{*}\right), \frac{1}{2}\left(d\left(T x^{*}, x^{*}\right)+d\left(x^{*}, T x^{*}\right)\right)\right\} \\
= & q d\left(T x^{*}, x^{*}\right) .
\end{aligned}
$$

Then

$$
T^{n} x^{*}=T x^{*}=x^{*}
$$

Then the fixed point is unique.

Remark 3. From the above Corollary 1 we can obtain a similar result by omitting the continuity of the operator $T$.

Further we will present a new generalization of a common fixed point theorem for Ćirić type operators in $b$-CVMS. The following result generalizes Theorem 1 in [14] in the sense that the space considered is more general than the one in the mentioned theorem.

Theorem 5. Let $(X, d)$ be a complete $b-C V M S$ with the coefficient $s \geq 1$ and $S, T: X \rightarrow X$ be two continuous mappings such that

$$
d(S x, T y) \precsim q \max \left\{d(x, y), \frac{d(x, S x) d(y, T y)}{1+d(x, y)}, \frac{d(x, T y) d(y, S x)}{1+d(x, y)}\right\},
$$

for all $x, y \in X$, where $0 \leq q<\frac{1}{s}$ and all elements on the right side are comparable to each other w.r.t. the partial order $\precsim$.

Then the pair $(S, T)$ have a unique common fixed point.

Proof. Let $x_{0}$ be arbitrary point in $X$ and define a sequence $\left\{x_{n}\right\}$ as follows

$$
x_{2 n+1}=S x_{2 n} \text { and } x_{2 n+2}=T x_{2 n+1}, n=0,1,2, \ldots
$$


Then, by (16) and (13), we get

$$
\begin{aligned}
d\left(x_{2 n+1}, x_{2 n+2}\right)= & d\left(S x_{2 n}, T x_{2 n+1}\right) \\
& \precsim q \max \left\{d\left(x_{2 n}, x_{2 n+1}\right), \frac{d\left(x_{2 n}, S x_{2 n}\right) d\left(x_{2 n+1}, T x_{2 n+1}\right)}{1+d\left(x_{2 n}, x_{2 n+1}\right)},\right. \\
& \left.\frac{d\left(x_{2 n}, T x_{2 n+1}\right) d\left(x_{2 n+1}, S x_{2 n}\right)}{1+d\left(x_{2 n}, x_{2 n+1}\right)}\right\} \\
& \precsim q \max \left\{d\left(x_{2 n}, x_{2 n+1}\right), \frac{d\left(x_{2 n}, x_{2 n+1}\right) d\left(x_{2 n+1}, x_{2 n+2}\right)}{1+d\left(x_{2 n}, x_{2 n+1}\right)},\right. \\
& \left.\frac{d\left(x_{2 n}, x_{2 n+2}\right) d\left(x_{2 n+1}, x_{2 n+1}\right)}{1+d\left(x_{2 n}, x_{2 n+1}\right)}\right\} \\
& \precsim q \max \left\{d\left(x_{2 n}, x_{2 n+1}\right), d\left(x_{2 n+1}, x_{2 n+2}\right)\right\} .
\end{aligned}
$$

Then $d\left(x_{2 n}, x_{2 n+1}\right) \precsim 1+1+d\left(x_{2 n}, x_{2 n+1}\right)$.

If $\max \left\{d\left(x_{2 n}, x_{2 n+1}\right), d\left(x_{2 n+1}, x_{2 n+2}\right)\right\}=d\left(x_{2 n+1}, x_{2 n+2}\right)$ then

$$
d\left(x_{2 n+1}, x_{2 n+2}\right) \precsim q d\left(x_{2 n+1}, x_{2 n+2}\right),
$$

This results $q \geq 1$, which is a contradiction. Therefore

$$
d\left(x_{2 n+1}, x_{2 n+2}\right) \precsim q d\left(x_{2 n}, x_{2 n+1}\right) .
$$

Similarly, we obtain that

$$
d\left(x_{2 n+2}, x_{2 n+3}\right) \precsim q d\left(x_{2 n+1}, x_{2 n+2}\right) .
$$

From (14) and (15) for all $n=0,1,2, \ldots$, we obtain

$$
d\left(x_{n+1}, x_{n+2}\right) \precsim q d\left(x_{n}, x_{n+1}\right) \precsim \ldots \precsim q^{n+1} d\left(x_{0}, x_{1}\right) .
$$

For $m, n \in \mathbb{N}$, with $m>n$ we obtain

$$
\begin{aligned}
d\left(x_{n}, x_{m}\right) & \precsim s\left[d\left(x_{n}, x_{n+1}\right)+d\left(x_{n+1}, x_{m}\right)\right] \\
& \precsim s\left(d\left(x_{n}, x_{n+1}\right)\right)+s^{2}\left[d\left(x_{n+1}, x_{n+2}\right)+d\left(x_{n+2}, x_{m}\right]\right. \\
& \precsim s\left(d\left(x_{n}, x_{n+1}\right)\right)+s^{2}\left(d\left(x_{n+1}, x_{n+2}\right)\right)+s^{3}\left(d\left(x_{n+2}, x_{n+3}\right)\right)+ \\
& +\ldots+s^{m-n-1}\left(d\left(x_{m-2}, x_{m-1}\right)\right)+s^{m-n}\left(d\left(x_{m-1}, x_{m}\right)\right) .
\end{aligned}
$$

By (2) we obtain

$$
\begin{aligned}
d\left(x_{n}, x_{m}\right) & \precsim s q^{n}\left(d\left(x_{0}, x_{1}\right)\right)+s^{2} q^{n+1}\left(d\left(x_{0}, x_{1}\right)\right)+s^{3} q^{n+2}\left(d\left(x_{0}, x_{1}\right)\right)+ \\
& +\ldots+s^{m-n-1} q^{m-2}\left(d\left(x_{0}, x_{1}\right)\right)+s^{m-n} q^{m-1}\left(d\left(x_{0}, x_{1}\right)\right)= \\
& =\sum_{i=1}^{m-n} s^{i} q^{i+n-1}\left(d\left(x_{0}, x_{1}\right)\right) .
\end{aligned}
$$

Therefore

$$
\begin{aligned}
\left|d\left(x_{n}, x_{m}\right)\right| & \precsim \sum_{i=1}^{m-n} s^{i+n-1} q^{i+n-1}\left|d\left(x_{0}, x_{1}\right)\right|=\sum_{t=n}^{m-1} s^{t} q^{t}\left|d\left(x_{0}, x_{1}\right)\right| \\
& \precsim \sum_{t=n}^{\infty}(s q)^{t}\left|d\left(x_{0}, x_{1}\right)\right|=\frac{(s q)^{n}}{1-s q}\left|d\left(x_{0}, x_{1}\right)\right| .
\end{aligned}
$$


Then, we obtain

$$
\left|d\left(x_{n}, x_{m}\right)\right| \precsim\left(\frac{(s q)^{n}}{1-s q}\right)\left|d\left(x_{0}, x_{1}\right)\right| \rightarrow 0 \text { as } n \rightarrow \infty .
$$

Therefore $\left\{x_{n}\right\}$ is a Cauchy sequence in $X$. Since $X$ is complete, then there exists $x^{*} \in X$ such that $d\left(x_{n}, x^{*}\right) \rightarrow 0$ as $n \rightarrow \infty$.

Since $S$ is continuous yields

$$
x^{*}=\lim _{n \rightarrow \infty} x_{2 n+2}=\lim _{n \rightarrow \infty} S x_{2 n+1}=S \lim _{n \rightarrow \infty} x_{2 n+1}=S x^{*} .
$$

Similarly, by the continuity of $T$, we get $x^{*}=T x^{*}$. Then the pair $(S, T)$ has a common fixed point.

For the uniqueness, assume that $y^{*} \in X$ is a second common fixed point of $S$ and $T$. Then

$$
\begin{aligned}
d\left(x^{*}, y^{*}\right) & =d\left(S x^{*}, T y^{*}\right) \\
& \precsim q \max \left\{d\left(x^{*}, y^{*}\right), \frac{d\left(x^{*}, S x^{*}\right) d\left(y^{*}, T y^{*}\right)}{1+d\left(x^{*}, y^{*}\right)}, \frac{d\left(x^{*}, T y^{*}\right) d\left(y^{*}, S x^{*}\right)}{1+d\left(x^{*}, y^{*}\right)}\right\} \\
& \precsim q d\left(x^{*}, y^{*}\right) .
\end{aligned}
$$

This implies $x^{*}=y^{*}$. Therefore $x^{*}$ is unique fixed point.

If we omit the continuity condition for the operators $S$ and $T$ in the previous theorem we have the following common fixed point result.

Theorem 6. Let $(X, d)$ be a complete $b-C V M S$ with the coefficient $s \geq 1$ and $S, T: X \rightarrow X$ two mappings such that

$$
d(S x, T y) \precsim q \max \left\{d(x, y), \frac{d(x, S x) d(y, T y)}{1+d(x, y)}, \frac{d(x, T y) d(y, S x)}{1+d(x, y)}\right\},
$$

for all $x, y \in X$, where $0 \leq q<\frac{1}{s}$ and all elements on the right side are comparable to each other w.r.t. the partial order $\precsim$.

Then the pair $(S, T)$ have a unique common fixed point.

Proof. Following same steps as in the proof of Theorem 5 we find a Cauchy sequence such that for $x^{*} \in X$ we have $\left\{x_{n}\right\} \rightarrow x^{*}$. Since $S$ and $T$ are not continuous we have $d\left(x^{*}, S x^{*}\right)=z>0$. Then we have the estimation

$$
\begin{aligned}
z & \precsim s\left[d\left(x^{*}, x_{2 k+2}\right)+d\left(S x^{*}, x_{2 k+2}\right)\right] \\
& \precsim s d\left(x^{*}, x_{2 k+2}\right)+s d\left(S x^{*}, T x_{2 k+1}\right) \\
& \precsim s d\left(x^{*}, x_{2 k+2}\right)+s \alpha \max \left\{d\left(x^{*}, x_{2 k+1}\right), \frac{d\left(x^{*}, S x^{*}\right) d\left(x_{2 k+1}, T x_{2 k+1}\right)}{1+d\left(x^{*}, x_{2 k+1}\right)},\right. \\
& \left.\frac{d\left(x^{*}, T x_{2 k+1}\right) d\left(x_{2 k+1}, S x^{*}\right)}{1+d\left(x^{*}, x_{2 k+1}\right)}\right\} \\
& \precsim s d\left(x^{*}, x_{2 k+2}\right)+s \alpha \max \left\{d\left(x^{*}, x_{2 k+1}\right), \frac{d\left(x^{*}, S x^{*}\right) d\left(x_{2 k+1}, x_{2 k+2}\right)}{1+d\left(x^{*}, x_{2 k+1}\right)},\right. \\
& \left.\frac{d\left(x^{*}, x_{2 k+2}\right) d\left(x_{2 k+1}, S x^{*}\right)}{1+d\left(x^{*}, x_{2 k+1}\right)}\right\} \\
& \precsim s d\left(x^{*}, x_{2 k+2}\right)+s q \max \{0,0, z\} \\
\precsim & s d\left(x^{*}, x_{2 k+2}\right)+s q z .
\end{aligned}
$$


This yields,

$$
|z| \leq s\left|d\left(x^{*}, x_{2 k+2}\right)\right|+s q|z| .
$$

Then results $q \geq 1$ which is a contradiction. Then $x^{*}=S x^{*}$. Similarly we get $x^{*}=T x^{*}$. Then the pair $(S, T)$ have a common fixed point.

For uniqueness of the common fixed point we use same steps as in the proof of Theorem 5 .

If we take $S=T$ in above theorems we get following fixed point result.

Theorem 7. Let $(X, d)$ be a complete $b$-CVMS with $s \geq 1$ be a given real number and $S: X \rightarrow X$ be a continuous mapping which satisfies

$$
d(S x, S y) \precsim q \max \left\{d(x, y), \frac{d(x, S x) d(y, S y)}{1+d(x, y)}, \frac{d(x, S y) d(y, S x)}{1+d(x, y)}\right\},
$$

for all $x, y \in X$, where $0 \leq q<\frac{1}{s}$ and all elements on the right side are comparable to each other w.r.t. the partial order $\precsim$. Then $S$ has a unique fixed point.

Remark 4. We can get a similar result without the continuity condition of the operator S.

Another fixed point result is the following.

Corollary 2. Let $(X, d)$ be a complete $b$-CVMS with $s \geq 1$ be a given real number and $S: X \rightarrow X$ be a continuous mapping which satisfies

$$
d\left(S^{n} x, S^{n} y\right) \precsim q \max \left\{d(x, y), \frac{d\left(x, S^{n} x\right) d\left(y, S^{n} y\right)}{1+d(x, y)}, \frac{d\left(x, S^{n} y\right) d\left(y, S^{n} x\right)}{1+d(x, y)}\right\},
$$

for all $x, y \in X$, where $0 \leq q<\frac{1}{s}, n \in \mathbb{N}$ and all elements on the right side are comparable to each other w.r.t. the partial order $\precsim$. Then $S$ has a unique fixed point.

Proof. By Theorem 7 we obtain an element $x^{*} \in X$ such that

$$
S^{n} x^{*}=x^{*}
$$

Then we have the following estimations

$$
\begin{aligned}
d\left(S x^{*}, x^{*}\right)=d\left(S S^{n} x^{*}, S^{n} x^{*}\right)= & d\left(S^{n} S x^{*}, S^{n} x^{*}\right) \\
\precsim & q \max \left\{d\left(S x^{*}, x^{*}\right), \frac{d\left(S x^{*}, S^{n} S x^{*}\right) d\left(x^{*}, S^{n} x^{*}\right)}{1+d\left(S x^{*}, x^{*}\right)},\right. \\
& \left.\frac{d\left(S x^{*}, S^{n} x^{*}\right) d\left(x^{*}, S^{n} S x^{*}\right)}{1+d\left(S x^{*}, x^{*}\right)}\right\} \\
& \precsim q \max \left\{d\left(S x^{*}, x^{*}\right), \frac{d\left(S x^{*}, S S^{n} x^{*}\right) d\left(x^{*}, S^{n} x^{*}\right)}{1+d\left(S x^{*}, x^{*}\right)},\right. \\
& \left.\frac{d\left(S x^{*}, S^{n} x^{*}\right) d\left(x^{*}, S S^{n} x^{*}\right)}{1+d\left(S x^{*}, x^{*}\right)}\right\} \\
= & q d\left(S x^{*}, x^{*}\right) .
\end{aligned}
$$

Then we obtain $S^{n} x^{*}=S x^{*}=x^{*}$. In this way we get that the fixed point is unique.

Remark 5. From the Corollary 2 we can obtain a similar result by omitting the continuity of the operator $S$.

Taking into account the above Remark, we give an illustrative example. 
Example 2. Let $X=\mathbb{C}$ be a set of complex number. Define $d: \mathbb{C} \times \mathbb{C} \rightarrow \mathbb{C}$, by

$$
d\left(z_{1}, z_{2}\right)=\left|x_{1}-x_{2}\right|^{2}+i\left|y_{1}-y_{2}\right|^{2},
$$

for all $z_{1}, z_{2} \in \mathbb{C}$, where $z_{1}=x_{1}+i y_{1}=\left(x_{1}, y_{1}\right)$ and $z_{2}=x_{2}+i y_{2}=\left(x_{2}, y_{2}\right)$. Obviously $(X, d)$ is a complete $b$-CVMS space with $s=2$.

Define $T: \mathbb{C} \rightarrow \mathbb{C}$, by

$$
T(z)=T(x+i y)= \begin{cases}0 & \text { If } x, y \in Q \\ 2+2 i & \text { If } x, y \in Q^{\prime} \\ 2 & \text { If } x \in Q^{\prime}, y \in Q \\ 2 i & \text { If } x \in Q, y \in Q^{\prime}\end{cases}
$$

where $Q$ is the set of rational numbers and $Q^{\prime}$ is the set of irrational numbers.

Now for $S=T$ and consider $x=\frac{1}{\sqrt{2}}$ and $y=0$.

$$
d(S x, T y)=d(T x, T y)=d\left(T \frac{1}{\sqrt{2}}, T 0\right)=d(2,0)=4 .
$$

and

$$
\begin{gathered}
d(x, y)=d\left(\frac{1}{\sqrt{2}}, 0\right)=\frac{1}{2} . \\
\frac{d(x, T x) d(y, T y)}{1+d(x, y)}=\frac{d\left(\frac{1}{\sqrt{2}}, T \frac{1}{\sqrt{2}}\right) d(0, T 0)}{1+d\left(\frac{1}{\sqrt{2}}, 0\right)}=\frac{d\left(\frac{1}{\sqrt{2}}, 2\right) d(0,0)}{1+d\left(\frac{1}{\sqrt{2}}, 0\right)}=0 . \\
\frac{d(x, T y) d(y, T x)}{1+d(x, y)}=\frac{d\left(\frac{1}{\sqrt{2}}, T 0\right) d\left(0, T \frac{1}{\sqrt{2}}\right)}{1+d\left(\frac{1}{\sqrt{2}}, 0\right)}=\frac{d\left(\frac{1}{\sqrt{2}}, 0\right) d(0,2)}{1+d\left(\frac{1}{\sqrt{2}}, 0\right)}=\frac{4}{3} .
\end{gathered}
$$

Then we have

$$
\begin{aligned}
d(T x, T y) & \precsim q \max \left\{d(x, y), \frac{d(x, T x) d(y, T y)}{1+d(x, y)}, \frac{d(x, T y) d(y, T x)}{1+d(x, y)}\right\} . \\
4 & \precsim q \max \left\{\frac{1}{2}, 0, \frac{4}{3}\right\} \precsim q \frac{4}{3} .
\end{aligned}
$$

Then we get a contradiction.

However, notice that $T^{n} z=0$ for $n>1$, we have

$$
d\left(T^{n} x, T^{n} y\right)=0 \precsim q \max \left\{d(x, y), \frac{d\left(x, T^{n} x\right) d\left(y, T^{n} y\right)}{1+d(x, y)}, \frac{d\left(x, T^{n} y\right) d\left(y, T^{n} x\right)}{1+d(x, y)}\right\},
$$

for all $x, y \in X$.

From the both Corollary 2 and Remark 5 we get a unique fixed point 0 of $T$.

\section{Applications on Integral Type Contractions}

In this section we apply our common fixed point results obtained above to prove common fixed points of certain contractions of integral type.

First let us recall the notion of altering distance function.

Definition 6. The function $\varphi:[0, \infty) \rightarrow[0, \infty)$ is called an altering distance function, if the following properties hold:

(i) $\varphi$ is continuous and nondecreasing,

(ii) $\varphi(t)=0$ if and only if $t=0$. 
Then, let us give the following definition.

Definition 7 . Let $\mathfrak{F}$ be the set of the function $f:[0, \infty) \rightarrow[0, \infty)$ that satisfies the following conditions:

(i) $f$ is Lebesgue Integrable on each compact subset of $[0, \infty)$;

(ii) $\int_{0}^{\varepsilon} f(t) d t>0$ for every $\varepsilon>0$.

Remark 6. It is easy to check that a mapping $\psi:[0, \infty) \rightarrow[0, \infty)$ defined by

$$
\psi(t)=\int_{0}^{\varepsilon} f(t) d t>0
$$

is an altering distance function.

Next we will give our first result of this section.

Theorem 8. Let $(X, d)$ be a complete $b$-CVMS with the coefficient $s \geq 1$ and $S, T: X \rightarrow X$ be two continuous mappings which satisfy

$$
\int_{0}^{d(S x, T y)} f(t) d t \precsim q \int_{0}^{M(x, y)} f(t) d t,
$$

for all $x, y \in X, 0 \leq q<\frac{1}{s}$ and $f \in \mathfrak{F}$ with

$$
M(x, y)=\max \left\{d(x, y), d(x, S x), d(y, T y), \frac{1}{2}(d(x, T y)+d(y, S x))\right\},
$$

where all elements of $M(x, y)$ are comparable to each other w.r.t. the partial order $\precsim$.

Then the pair $(S, T)$ has a unique common fixed point.

Proof. Using Theorem 2 for $\psi(t)=\int_{0}^{t} f(u) d u$ we get the conclusion.

Remark 7. We get a similar result without the continuity condition of the mappings $S$ and $T$.

For $S=T$ we get another two fixed point of integral type results with continuity condition of the operator $T$ and without it.

Theorem 9. Let $(X, d)$ be a complete $b$-CVMS with the coefficient $s \geq 1$ and $T: X \rightarrow X$ be $a$ continuous mapping which satisfies the following condition

$$
\int_{0}^{d(T x, T y)} f(t) d t \precsim q \int_{0}^{N(x, y)} f(t) d t
$$

for all $x, y \in X, 0 \leq q<\frac{1}{s}$ and $f \in \mathfrak{F}$ with

$$
N(x, y)=\max \left\{d(x, y), d(x, T x), d(y, T y), \frac{1}{2}(d(x, T y)+d(y, T x))\right\},
$$

where all elements of $N(x, y)$ are comparable to each other w.r.t. the partial order $\precsim$.

Then $T$ has a unique fixed point.

Proof. Using Theorem 4 for $\psi(t)=\int_{0}^{t} f(u) d u$ we get the conclusion.

For the generalization of the Ćirić type contraction we get the following common fixed point integral type result. 
Theorem 10. Let $(X, d)$ be a complete $b$-CVMS with the coefficient $s \geq 1$ and $S, T: X \rightarrow X$ be two continuous mappings which satisfy

$$
\int_{0}^{d(S x, T y)} f(t) d t \precsim q \int_{0}^{P(x, y)} f(t) d t,
$$

for all $x, y \in X, 0 \leq q<\frac{1}{s}$ and $f \in \mathfrak{F}$ with

$$
P(x, y)=\max \left\{d(x, y), \frac{d(x, S x) d(y, T y)}{1+d(x, y)}, \frac{d(x, T y) d(y, S x)}{1+d(x, y)}\right\},
$$

where all elements of $P(x, y)$ are comparable to each other w.r.t. the partial order $\precsim$.

Then the pair $(S, T)$ has a unique common fixed point.

Proof. Using Theorem 5 for $\psi(t)=\int_{0}^{t} f(u) d u$ we get the conclusion.

Remark 8. We get a similar result without the continuity condition of the mappings $S$ and $T$.

For $S=T$ we get another two fixed point of integral type results for this new type of Ćirić contraction with continuity condition of the operator $T$ and without it.

Theorem 11. Let $(X, d)$ be a complete $b$-CVMS with the coefficient $s \geq 1$ and $T: X \rightarrow X$ be $a$ continuous mapping which satisfies

$$
\int_{0}^{d(T x, T y)} f(t) d t \precsim q \int_{0}^{Q(x, y)} f(t) d t,
$$

for all $x, y \in X, 0 \leq q<\frac{1}{s}$ and $f \in \mathfrak{F}$ with

$$
Q(x, y)=\max \left\{d(x, y), \frac{d(x, T x) d(y, T y)}{1+d(x, y)}, \frac{d(x, T y) d(y, T x)}{1+d(x, y)}\right\},
$$

where all elements of $Q(x, y)$ are comparable to each other w.r.t. the partial order $\precsim$.

Then $T$ has a unique fixed point.

Proof. By Theorem 7 for $\psi(t)=\int_{0}^{t} f(u) d u$ we get the conclusion.

\section{Application on an Urysohn Type Integral Equation System}

Through the last few years the Banach contraction principle was a reference for many researchers in the field of nonlinear analysis and it was a strong tool in establishment to the existence of a unique solution for a nonlinear integral equation. In this section we apply our common fixed point results obtained above to prove the existence of a common solution of an integral type system. The starting point in considering the following application was the work of W. Sintunavarat et. al [26].

Let us consider the following system of Urysohn type integral equations.

$$
\left\{\begin{array}{l}
x(t)=f(t)+\int_{a}^{b} P_{1}(t, s, x(s)) d s \\
y(t)=f(t)+\int_{a}^{b} P_{2}(t, s, y(s)) d s
\end{array} .\right.
$$

where,

(i) $x(t)$ and $y(t)$ are unknown variables for each $t \in[a, b], a>0$,

(ii) $f(t)$ is the deterministic free term defined for $t \in[a, b]$, 
(iii) $P_{1}(t, s)$ and $P_{2}(t, s)$ are deterministic kernels defined for $t, s \in[a, b]$. Let $X=\left(C[a, b], \mathbb{R}^{n}\right), \tau>0$ and $d: X \times X \rightarrow \mathbb{R}^{n}$ defined by

$$
d(x, y)=\|x(t)-y(t)\|_{\infty}=\sup _{t \in[a, b]}|x(t)-y(t)|^{2} \sqrt{1+\tau^{2}} e^{i \tan ^{-1} \tau},
$$

for all $x, y \in X, i=\sqrt{-1} \in \mathbb{C}$

Obviously $\left(C[a, b], \mathbb{R}^{n},\|\cdot\|_{\infty}\right)$ is a complete $b$-CVMS with the constant $s=2$.

Further let us consider a Urysohn type integral system as (21) under the following conditions:

$\left(u_{1}\right) f(t) \in X$,

$\left(u_{2}\right) P_{1}, P_{2}:[a, b] \times[a, b] \times \mathbb{R}^{n} \rightarrow \mathbb{R}^{n}$ are continuous functions satisfying

$$
\left|P_{1}(t, s, u(s))-P_{2}(t, s, v(s))\right| \precsim \frac{1}{\sqrt{(b-a) e^{\tau}}} M(u, v),
$$

where,

$$
M(u, v)=\max \left\{d(u, v), d(u, T u), d(v, T v), \frac{1}{2}(d(u, T v)+d(v, T u))\right\} .
$$

In this section we apply Theorem 2 to prove the existence of a unique solution of the system (21). Then our main theorem of this section is the following.

Theorem 12. $\left(C[a, b], \mathbb{R}^{n},\|\cdot\|_{\infty}\right)$ be a complete complex valued metric space, then the system (21) under the conditions $\left(u_{1}\right)$ and $\left(u_{2}\right)$ has a unique common solution.

Proof. For $x, y \in\left(C[a, b], \mathbb{R}^{n}\right)$ and $t \in[a, b]$, we define the continuous mappings $S, T:$ $X \rightarrow X$ by

$$
\begin{aligned}
& S x(t)=f(t)+\int_{a}^{b} P_{1}(t, s, x(s)) d s, \\
& T y(t)=f(t)+\int_{a}^{b} P_{2}(t, s, y(s)) d s .
\end{aligned}
$$

Then we have

$$
\begin{aligned}
|S x(t)-T y(t)|^{2} & =\int_{a}^{b}\left|P_{1}(t, s, x(s))-P_{2}(t, s, y(s))\right|^{2} d s \\
& \precsim \int_{a}^{b} \frac{1}{(b-a) e^{\tau}}|M(x, y)|^{2} d s \\
& =\frac{1}{(b-a) e^{\tau}} \int_{a}^{b} \frac{e^{-i \tan ^{-1} \tau}}{\sqrt{1+\tau^{2}}}|M(x, y)|^{2} \sqrt{1+\tau^{2}} e^{i \tan ^{-1} \tau} d s \\
& \precsim \frac{1}{(b-a) e^{\tau}} \frac{e^{-i \tan ^{-1} \tau}}{\sqrt{1+\tau^{2}}}\|M(x, y)\|_{\infty} \int_{a}^{b} d s \\
& =\frac{1}{e^{\tau}} \frac{e^{-i \tan ^{-1} \tau}}{\sqrt{1+\tau^{2}}}\|M(x, y)\|_{\infty} .
\end{aligned}
$$

Then we get

$$
|S x(t)-T y(t)|^{2} \sqrt{1+\tau^{2}} e^{i \tan ^{-1} \tau} \precsim \frac{1}{e^{\tau}}\|M(x, y)\|_{\infty}
$$

or, equivalently

$$
\|S x(t)-T y(t)\|_{\infty} \precsim \frac{1}{e^{\tau}}\|M(x, y)\|_{\infty} .
$$

Then we have

$$
d(S x, T y) \precsim \gamma M(x, y) .
$$


Then the conditions of Theorem 2 are satisfied for $0<\gamma=\frac{1}{e^{\tau}}<1$, with $\tau>0$. Therefore the system (21) has a unique common solution on $X$.

In order to sustain our results we present the following example.

Example 3. Let $X=C([0,1], \mathbb{R})$ and the following nonlinear integral equation as the form:

$$
\left\{\begin{array}{l}
x(t)=e^{2 i t}+\int_{0}^{1}\left(\frac{e^{-\frac{1}{2}}}{2\left(t+\frac{i s}{1+i s}+x(s)\right)}\right) d s \\
y(t)=e^{2 i t}+\int_{0}^{1}\left(\frac{e^{-\frac{1}{2}}}{2\left(t+\frac{i s}{1+i s}+y(s)\right)}\right) d s
\end{array}\right.
$$

System (22) is a particular case of system (21), where $f(t)=e^{2 i t}$ and

$$
P_{i}\left(t, s, v_{i}(s)\right)=\left(\frac{e^{-\frac{1}{2}}}{2\left(t+\frac{i s}{1+i s}+v_{i}(s)\right)}\right), i=1,2 .
$$

It's obvious that $\left(u_{1}\right)$ is satisfied, for $\left(u_{2}\right)$, we get

$$
\begin{aligned}
\left|P_{1}(t, s, x(s))-P_{2}(t, s, y(s))\right|^{2} & =\frac{1}{4} e^{-1}\left|\frac{x(s)-y(s)}{\left(t+\frac{i s}{1+i s}+x(s)\right)\left(t+\frac{i s}{1+i s}+y(s)\right)}\right|^{2} \\
& \precsim \frac{1}{4} e^{-1}|x(s)-y(s)|^{2} .
\end{aligned}
$$

Therefore, $\left(u_{2}\right)$ holds with $\gamma=\frac{1}{4} e^{-1}<1$ and $M(x, y)=|x(s)-y(s)|^{2}$. By Theorem 12, the system (22) has a unique solution.

\section{Conclusions}

Fixed point theory is a powerful tool for proving the existence and the uniqueness of different type of equations. In the first part of this paper it is proved, in the setting of a complex valued $b$-metric space, the existence and uniqueness of a common fixed point for two singlevalued mappings that satisfy a Ćirić type contraction condition. The section Application sustains the obtain results in the sense that the main results will be applied in order to obtain common fixed points of some contractions of integral type. The results obtained in this paper exetend and generalize some new results in the literature.

It is an open problem to obtain some similar results for operators that satisfy some other contraction conditions. Another open problem is to obtain similar results for the case of multivalued operators.

Author Contributions: Conceptualization, M.S.R.C., L.G. and M.F.B.; methodology, N.S.; software, M.S.A.; validation, M.S.R.C., N.S. and M.F.B.; formal analysis, L.G.; investigation, N.S.; resources, M.F.B.; writing—original draft preparation, M.S.A.; writing—review and editing, L.G.; visualization, M.F.B.; supervision, M.S.R.C.; project administration, N.S.; funding acquisition, M.F.B. All authors have read and agreed to the published version of the manuscript.

Funding: This research received no external funding.

Informed Consent Statement: Not applicable.

Data Availability Statement: Not applicable.

Acknowledgments: The authors want to thank to the reviewers for them valuable remarks and recommendations.

Conflicts of Interest: The authors declare no conflict of interest. 


\section{References}

1. Arandjelović, I.; Radenovixcx, Z.; Radenovixcx, S. Boyd-Wong-type common fixed point results in cone metric spaces. Appl. Math. Comput. 2011, 217, 7167-7171. [CrossRef]

2. Bhatt, S.; Chaukiyal, S.; Dimri, R.C. A common fixed point theorem for weakly compatible maps in complex valued metric spaces. Int. J. Math. Sci. Appl. 2011, 1, 1385-1389.

3. Boyd, D.W.; Wong, J.S.W. On nonlinear contractions. Proc. Am. Math. Soc. 1969, 20, 458464. [CrossRef]

4. Dugundji, J.; Granas, A. Fixed Point Theory; Monograe Matematycne: Warsazawa, Poland, 1982.

5. Tarafdar, E. An approach to fixed-point theorems on uniform spaces. Trans. Am. Math. Soc. 1974, 191, 209-225. [CrossRef]

6. Bakhtin, I.A. The contraction mapping principle in quasimetric spaces. Funct. Anal. Unianowsk Gos. Ped. Inst. $1989,30,26-37$.

7. Czerwik, S. Contraction mappings in b-metric spaces. Acta Math. Inform. Univ. Ostrav. 1993, 1, 5-11.

8. Czerwik, S. Nonlinear set-valued contraction mappings in b-metric spaces. Atti Sem. Mat. Univ. Modena 1998, 46, $263-276$.

9. Roshan, J.; Parvaneh, V.; Sedghi, S.; Shobkolaei, N.; Shatanawai, W. Common fix points of almost generalized $(\psi, \varphi)_{s}$-contractive mapping in ordered $b$-metric spaces. Fixed Point Theory Appl. 2013, 2013, 159. [CrossRef]

10. Saleem, N.; Iqbal, I.; Iqbal, B.; Radenovíc, S. Coincidence and fixed points of multivalued F-contractions in generalized metric space with application. J. Fixed Point Theory Appl. 2020, 81. [CrossRef]

11. Saleem, N.; Vujaković, J.; Baloch, W.U.; Radenović, S. Coincidence point results for multivalued Suzuki type mappings using $\theta$-contraction in $b$-metric spaces. Mathematics 2019, 7, 10-17. [CrossRef]

12. Azam, A.; Fisher, B.; Khan, M. Common fixed point theorems in complex valued metric spaces. Numer. Funct. Anal. Optim. 2011, 32, 243-253. [CrossRef]

13. Chandok, S.; Kumar, D. Some common fixed point results for rational type contraction mappings in complex valued metric spaces. J. Oper. 2013, 2013, 1-7. [CrossRef]

14. Hammad, H.A.; De la Sen, M. Analytical solution of Urysohn integral equations by fixed point technique in complex valued metric spaces. Mathematics 2019, 7, 852. [CrossRef]

15. Mitra, T. A common coupled fixed point result in complex valued metric space for two mappings. Int. J. Curr. Res. 2015, 7, 19555-19559.

16. Mukheimer, A.A. Some common fixed point theorems in complex valued $b$-metric spaces. Sci. World J. 2014, 10, 1-6. [CrossRef]

17. Verma, R.K.; Pathak, H.K. Common Fixed Point Theorems Using Property (E.A) in Complex-Valued Metric Spaces. Thai J. Math 2013, 11, 347-355.

18. Belhenniche, A.; Benahmed, S.; Guran, L. Existence of a solution for integral Urysohn type equations system via fixed points technique in complex valued extended $b$-metric spaces. J. Prime Res. Math. 2020, 16, 109-122.

19. Öztürk, M.; Kaplan, N. Common fixed points of $f$-contraction mappings in complex valued metric spaces. Math. Sci. 2014, 8, 129. [CrossRef]

20. Singh, N.; Singh, D.; Badal, A.; Joshi, V. Fixed point theorems in complex valued metric spaces. J. Egypt. Math. Soc. 2016, 24, 402-409. [CrossRef]

21. Rao, K.P.R.; Swamy, P.R.; Prasad, J.R. A common fixed point theorem in complex valued $b$-metric spaces. Bull. Math. Stat. Res. 2013, 1, 1-8.

22. Singh, D.; Chauhan, O.P.; Singh, N.; Joshi, V. Common fixed Point theorems in complex valued b-metric spaces. J. Math. Comput. Sci. 2015, 5, 412-429.

23. Kirk, W.A.; Sims, B. Handbook of Metric Fixed Point Theory; Kluwer Academic Publishers: Iowa City, IA, USA; Newcastle, WY, USA, 2001.

24. Ćirić, L. Generalized contractions and fixed point theorems. Publ. Inst. Math. Beogr. 1971, 12, $19-26$.

25. Miculescu, R.; Mihail, A. New fixed point theorems for set-valued contractions in b-metric spaces. J. Fixed Point Theory Appl. 2017, 19, 2153-2163. [CrossRef]

26. Sintunavarat, W.; Cho, Y.J.; Kumam, P. Urysohn integral equations approach by common fixed points in complex-valued metric spaces. Adv. Differ. Equ. 2013, 2013, 49. [CrossRef] 